\title{
RMetS
}

Royal Meteorological Society

\section{Relaxing the Tropics to an 'observed' state: analysis using a simple baroclinic model}

\author{
Brian Hoskins, ${ }^{\text {a* Ricardo Fonseca, }{ }^{\mathrm{b}} \text { Mike Blackburn }{ }^{\mathrm{c}} \text { and Thomas Jung }}{ }^{\mathrm{d}}$ \\ ${ }^{a}$ Grantham Institute for Climate Change, Imperial College \\ ${ }^{\mathrm{b}}$ University of Reading, Department of Meteorology \\ ${ }^{c}$ National Centre for Atmospheric Science, University of Reading \\ ${ }^{\mathrm{d}}$ Alfred-Wegener Institute for Polar and Marine Research (AWI), University of Bremen \\ ${ }^{*}$ Correspondence to: B. Hoskins, Grantham Institute for Climate Change, Imperial College London, Exhibition Road, \\ South Kesington, London SW7 2AZ, UK. E-mail: b.hoskins@imperial.ac.uk
}

The technique of relaxation of the tropical atmosphere towards an analysis in a month-season forecast model has previously been successfully exploited in a number of contexts. Here it is shown that when tropical relaxation is used to investigate the possible origin of the observed anomalies in June-July 2007, a simple dynamical model is able to reproduce the observed component of the pattern of anomalies given by an ensemble of ECMWF forecast runs. Following this result, the simple model is used for a range of experiments on time-scales of relaxation, variables and regions relaxed based on a control model run with equatorial heating in a zonal flow.

A theory based on scale analysis for the large-scale tropics is used to interpret the results. Typical relationships between scales are determined from the basic equations, and for a specified diabatic heating a chain of deductions for determining the dependent variables is derived. Different critical time-scales are found for tropical relaxation of different dependent variables to be effective. Vorticity has the longest critical time-scale, typically 1.2 days. For temperature and divergence, the timescales are 10 hours and 3 hours, respectively. However not all the tropical fields, in particular the vertical motion, are reproduced correctly by the model unless divergence is heavily damped. To obtain the correct extra-tropical fields, it is crucial to have the correct rotational flow in the subtropics to initiate the Rossby wave propagation from there. It is sufficient to relax vorticity or temperature on a time-scale comparable or less than their critical time-scales to obtain this. However if the divergent advection of vorticity is important in the Rossby Wave Source then strong relaxation of divergence is required to accurately represent the tropical forcing of Rossby waves. Copyright (C) 2012 Royal Meteorological Society

Key Words: large-scale tropical dynamics; tropical relaxation; critical time-scales

Received 12 August 2011; Revised 6 December 2011; Accepted 13 December 2011; Published online in Wiley Online Library

Citation: Hoskins B, Fonseca R, Blackburn M, Jung T. 2012. Relaxing the Tropics to an 'observed' state: analysis using a simple baroclinic model. Q. J. R. Meteorol. Soc. DOI:10.1002/qj.1881

1. Introduction

The technique of relaxation of the tropical atmosphere back to an analysed state has been used for the past two decades in global weather forecast models in order to reveal the global dependence on poorly captured variability in the tropics (Ferranti et al., 1990) and the importance for mean errors throughout the globe on those in the tropics (Klinker, 


\section{B. Hoskins et al.}

1990). It has also been used to investigate the possible tropical origin of extratropical extended-range forecast error (Jung et al., 2010a) and of monthly-seasonal anomalies in higher latitudes (Jung et al., 2010b), as well as to investigate the impact of the extratropics on the tropics (Vitart and Jung, 2010). Pohl and Douville (2011) also used this technique to investigate the impact of atmospheric behaviour outside the West African region on the summer monsoon there. In addition, Jung (2011) has found that the techniques of tropical relaxation and running a full data assimilation incorporating data only from the tropical region give very similar results.

An example of the use of tropical relaxation in an investigation of the importance of the tropical state in the anomalous June-July 2007 period is given in Figure 1. This period, which was analysed by Blackburn et al. (2008), was very wet in the UK and dry over southeastern Europe. The observed $500 \mathrm{hPa}$ geopotential anomalies (Figure 1(a)) show low heights centred to the southwest of the UK and high heights over southeastern Europe. There are also prominent wave trains across the North Pacific with a low over the Gulf of Alaska, and across the Southern Ocean. Figure 1(b) gives the average field resulting from an ensemble of forecasts of the T159, L60 ECMWF model, with a set-up as in Jung et al. (2008). Here each field in the tropical atmosphere is relaxed back towards the analysed state with a relaxation time-scale of $10 \mathrm{~h}$, the analyses being updated every $6 \mathrm{~h}$. The wave trains across the North Pacific and that in the Southern Ocean are represented very well, showing the importance of the presence of the tropics. However, this is not the case for the low near the UK and the high over southeast Europe, suggesting the role of extratropical processes here.

The tropical relaxation technique has been developed in a practical context and, as such, is clearly very useful. However, there is a lack of a theoretical basis to guide its implementation. Also, there is the question of whether it would be valuable to apply it in simpler models that could provide insight and guidance in experimentation with complex models. These comments provide the motivation for the present paper.

The simpler model to be used here will be described in detail in section 2. It is a T42 L15 adiabatic model with linear damping terms and also with added forcing terms, which act to maintain the climatological flow. The model is run once for 30 days with the tropics relaxed back to the mean anomaly for June-July 2007, and the $500 \mathrm{hPa}$ height field for day 30 is given in Figure 1(c). Given the simplicity of the model, the results are remarkable in their simulation of the observed anomalies and their similarity with the ECMWF ensemble results. Over the North Pacific and the Southern Oceans the day-30 anomalies look at least as good as those given by the ensemble with the full forecast system. The low near the UK is much better positioned in longitude, although it is still too far north, and the high over southeastern Europe is again absent. However, it should be noted that the contour interval for the simpler model result is half that for both the observations and the ECMWF ensemble. This means that the amplitudes of the Northern Hemisphere anomalies are about half those observed and simulated in the ECMWF system. This may be due to lack of physical processes, particularly latent heat release, and transient eddy flux convergences that may feedback positively on to the anomalies. A number of conclusions are suggested from these results.

1 In June-July 2007 the correctly reproduced extratropical anomalies appear to be basically due to Rossby wave propagation from the tropics, the only process likely to produce them in the simpler model;

2 The range of processes represented in the forecast model and its set-up, and not in the simpler model and its set-up, appear to lead merely to an amplification of the Northern Hemisphere signal;

3 It is of interest to investigate more fully the tropical relaxation technique using this simpler model.

(a)

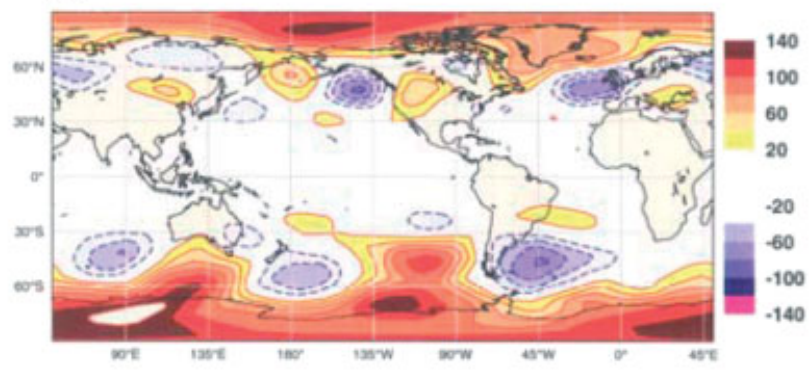

(b)

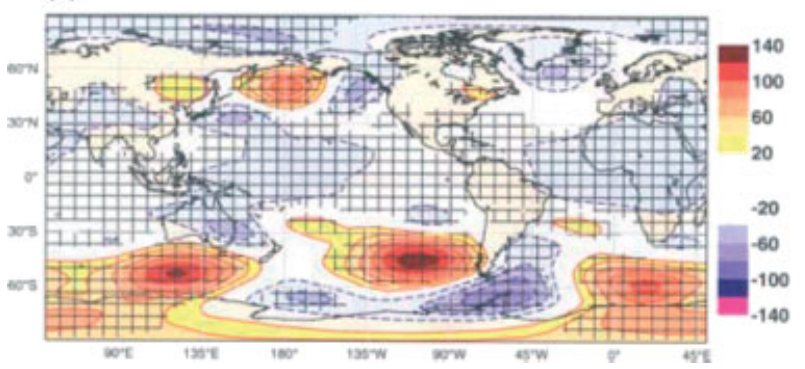

(c)

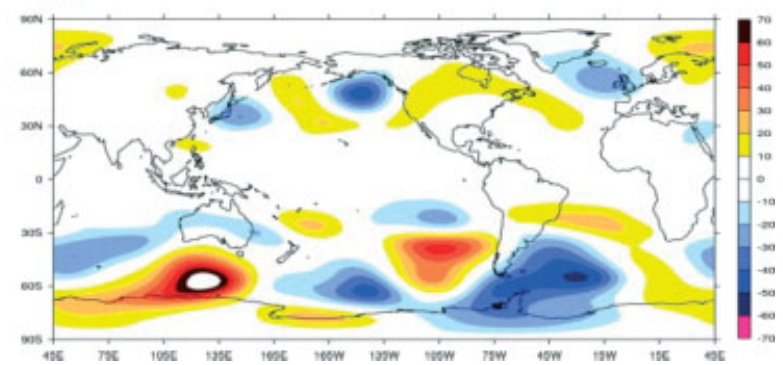

Figure 1. Geopotential height anomalies at $500 \mathrm{hPa}$. (a) Observed averaged over June-July 2007 and obtained by relaxing the wider tropics ( $30^{\circ} \mathrm{N}-30^{\circ} \mathrm{S}$ ) using the (a) ECMWF model and (c) IGCM. In (b) statistically significant anomalies at the 95\% confidence level are hatched and the contour interval is $20 \mathrm{~m}$, whereas in (c) it is $10 \mathrm{~m}$. 
Some details of this model are given in section 2 . In section 3 it is used for an experiment with an imposed idealized tropical heat source, and this is used to provide control data for relaxation experiments for which the correct answer is known. First the sensitivity of the results to the time-scale for tropical relaxation is examined. Then the dependence on the variable used for the relaxation and also the importance of the deep tropical and subtropical 'observed' data are tested. To provide a basis for understanding these results, a theory based on scale analysis is developed and exploited in section 4. Some concluding comments are made in section 5 .

\section{Experimental set-up}

The experiments described in this paper were performed with a version of the Reading spectral model (Hoskins and Simmons, 1975). The model is run at a T42 horizontal truncation and 15 levels with some concentration in the tropopause region as in Jin and Hoskins (1995). On the two lowest levels $(\sigma=\mathbf{0 . 9 6 7 , 0 . 8 8 7})$ a differential damping scheme is used whereby the winds are damped on timescales of 4 days and 3/2 days over the oceans and 1 day and 3/8 day over land, respectively, as in Rodwell and Hoskins (1995). In the thermodynamic equation Newtonian cooling is included with the model temperature relaxed towards a specified temperature distribution, generally with a timescale of 25 days decreasing gradually to 1 day at the top model level and 2 days at the lowest model level, following Jin and Hoskins (1995). This is used to damp thermal anomalies about a basic state. A $\nabla^{4}$ hyperdiffusion, with a time-scale of $6 \mathrm{~h}$ on the smallest resolved horizontal scale, is applied to the vorticity, divergence and temperature. Vertical diffusion is also included with a mixing coefficient of $5 \mathrm{~m}^{2} \mathrm{~s}^{-1}$ applied to the perturbation vorticity, divergence and temperature fields.

As in Jin and Hoskins (1995) the model is run by maintaining a three-dimensional climatology: a constant forcing is applied that maintains the basic flow of interest in the absence of diabatic or frictional sources. In the experiments described in section 3 the basic state is the zonal-mean December-February (hereafter DJF) ERAInterim (1989-2008) climatology. Various forms of the Reading spectral model have been used and often with some representation of the physical processes they have been referred to as IGCM (Intermediate GCM). However, to emphasize its dynamical core essence, the model will be referred to here as the dynamical circulation model (DCM). Fields that can be relaxed in the DCM are the prognostic variables: absolute vorticity, horizontal divergence, temperature and the logarithm of the surface pressure.

To perform a relaxation, a term of the form $-\lambda\left(X-X_{\text {ref }}\right)$ is added to the model equations, so that the model state vector $X$ is relaxed towards a reference field $X_{\text {ref. }}$. The strength of the relaxation is determined by the inverse time-scale $\lambda$ which can be a function of the variable, horizontal/vertical region or spatial scale. For a model timestep of $1 \mathrm{~h}$, a value of $\lambda=0.1$ means that at each hour the model is 'corrected' using $10 \%$ of the departure of $X$ from $X_{\text {ref. Different relaxation time-scales are considered }}$ with the standard being $10 \mathrm{~h}$, the time-scale generally used by ECMWF in their experiments. The relaxation is to be applied only in specific regions of the sphere and therefore the term is calculated in grid point space rather than spectral space.

\section{Idealized heating experiments}

The experiments discussed in this section were performed with the aim of testing the ability of relaxation to reproduce the local and remote responses to an idealized forcing, the sensitivity to the relaxation time-scale and variable relaxed, and the relative importance of the deep Tropics and Subtropics as the relaxation region. A control experiment was first performed in which the model was forced with a specified idealized equatorial heating anomaly and with the zonal-mean DJF climatology as the basic state. The zonal average of the heating was removed (uniform cooling elsewhere in the same latitude) and the vertical profile was taken from Jin and Hoskins (1995) with a maximum heating rate of $5 \mathrm{~K} \mathrm{day}^{-1}$ at $\sigma \sim 0.4$. It is found that the global large-scale pattern in this control experiment is set up by days 15 to 20 , and that it subsequently changes very little up to day 40, so that day 20-30 averages and the day 30 fields are almost indistinguishable. Baroclinic instability starts to become noticeable in the v-field after day 50 and dominates the results after day 60 . The response at day 30 is illustrated in Figure 2 (the heating, shown by the black ellipse, extends approximately from $15^{\circ} \mathrm{S}$ to $15^{\circ} \mathrm{N}$ ). The geography is used as a background to help interpretation: the only role played in the model is the enhanced drag over land.

In the control experiment, the $200 \mathrm{hPa}$ streamfunction anomalies, Figure 2(a), show a standard Gill-Matsuno response with a pair of anticyclones to the west of the forcing and a pair of cyclones to the east. There is also a wave train across the North Pacific into North America with a trough over Alaska and northwestern Canada and a ridge over northeastern USA. A similar pattern is obtained in the temperature anomalies, Figure 2(c), with upper tropospheric ridges corresponding to mid-tropospheric warming and upper tropospheric troughs to mid-tropospheric cooling corresponding to the first baroclinic mode in the tropics and equivalent barotropic structures away from there. The $400 \mathrm{hPa}$ omega anomalies, Figure 2(b), show the local ascent in the heating region and emphasize a wave train across the northern subtropical Pacific.

In Figure 3 the day 30 responses are shown when, in place of the equatorial heating, the model is relaxed in the tropics towards the control state reached at day 30 while imposing forcing that would maintain the basic zonalmean DJF climatology. Results for relaxation time-scales on all variables of $1 \mathrm{~h}, 10 \mathrm{~h}, 1$ day and 5 days are given. The pattern of streamfunction anomalies, and therefore the rotational component of the flow, is well reproduced in each and the amplitude is also good for relaxation timescales up to 1 day. However, the response weakens with increasing relaxation time-scale: e.g. the maximum to the west of the heating decreases from the 'correct' value of about $12 \times 10^{6} \mathrm{~m}^{2} \mathrm{~s}^{-1}$ for time-scales of $1 \mathrm{~h}$ and $10 \mathrm{~h}$ to $11 \times$ $10^{6} \mathrm{~m}^{2} \mathrm{~s}^{-1}$ for a time-scale of 1 day and $6 \times 10^{6} \mathrm{~m}^{2} \mathrm{~s}^{-1}$ for a relaxation time-scale of 5 days. For a time-scale of 5 days, the amplitude of the response is therefore about half the one in the original experiment, although the phase is still well reproduced.

The results for omega, and therefore for the divergent component of the flow, show a different picture: the local 


\section{B. Hoskins et al.}

(a)

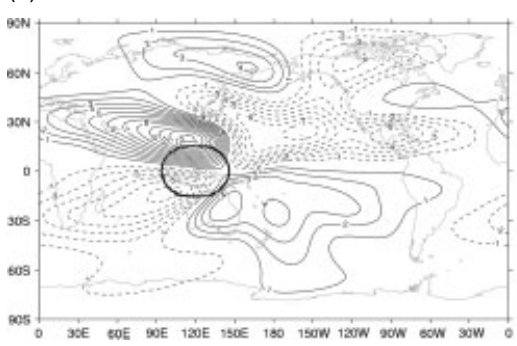

(b)

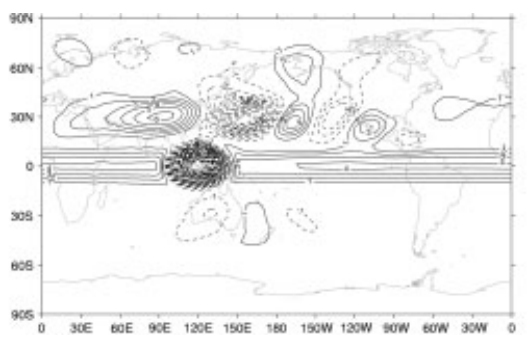

(c)

$\mathrm{T}^{\prime}{ }_{500 \mathrm{hPa}}$

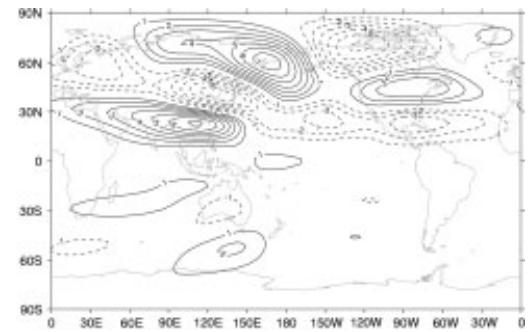

Figure 2. (a) Streamfunction anomalies at $200 \mathrm{hPa}$ (units of $10^{6} \mathrm{~m}^{2} \mathrm{~s}^{-1}$ ), (b) omega anomalies at $400 \mathrm{hPa}$ (units of $1 \times 10^{-1}$ Pas ${ }^{-1}$ ) and (c) temperature anomalies at $500 \mathrm{hPa}$ (units of $2 \times 10^{-1} \mathrm{~K}$ ) at day 30 in response to a heating over Indonesia (shown by the black ellipsis in (a)) with the ERA-Interim DJF zonal-mean climatology as basic state. Positive values are represented by solid lines and negative values by dashed lines. The zero line has been removed.

(a)

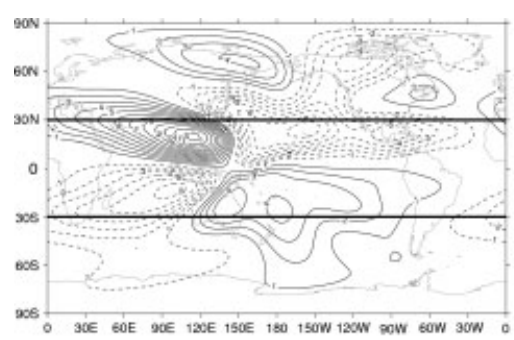

(b)

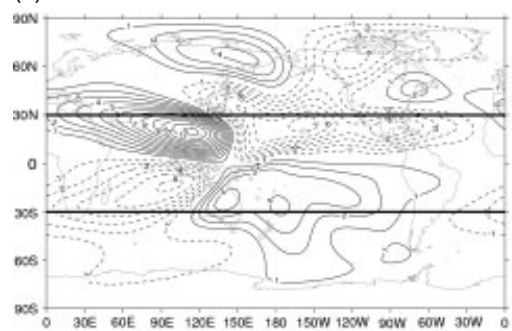

(c)

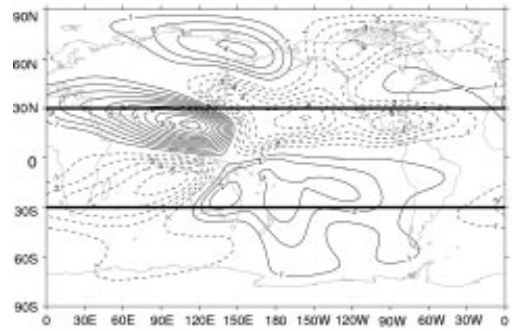

(d)

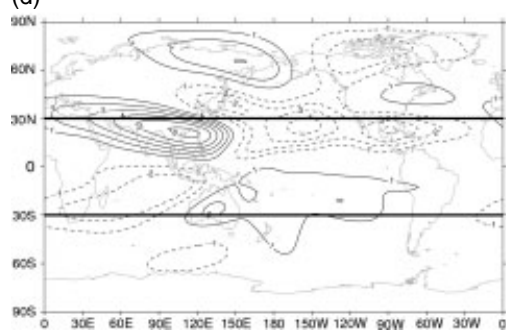

(e)

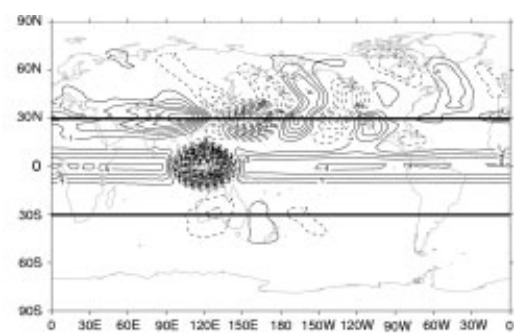

(f)

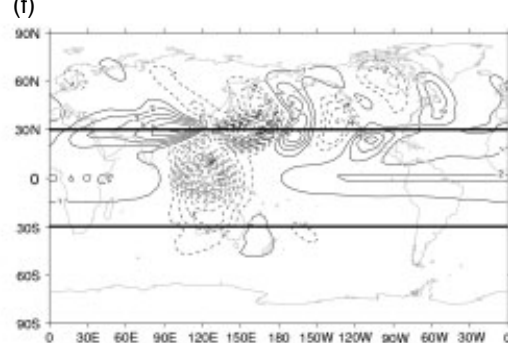

(g)

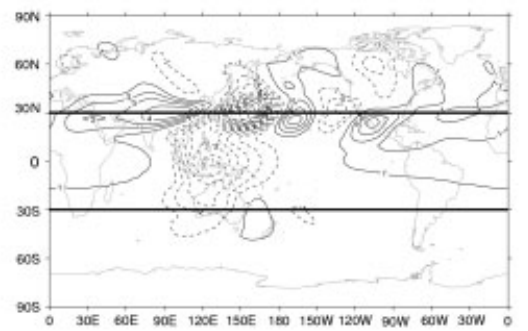

(h)

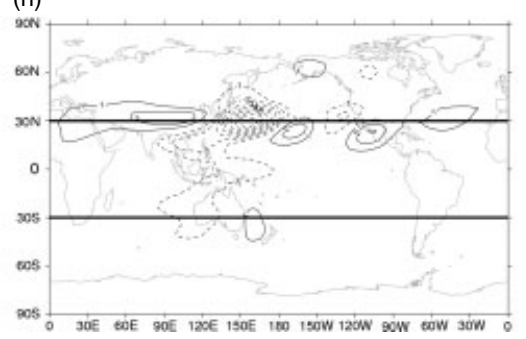

$\mathrm{T}_{500 \mathrm{hPa}}$

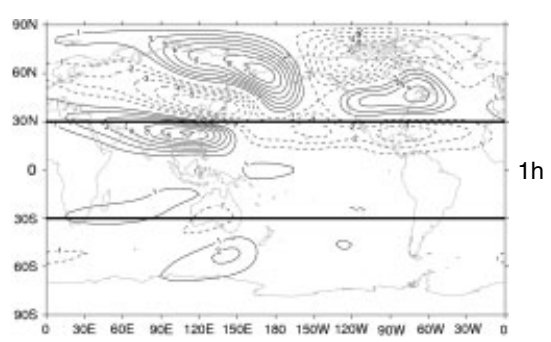

(j)

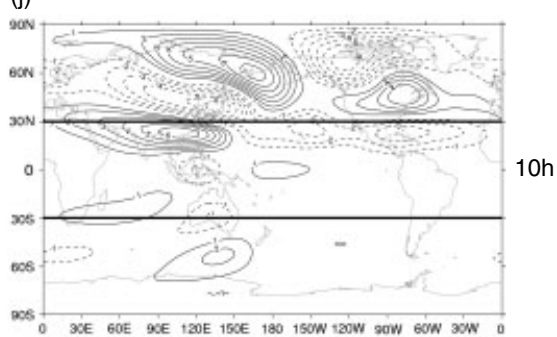

(k)

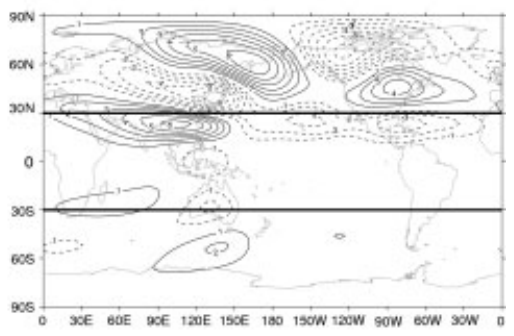

(I)

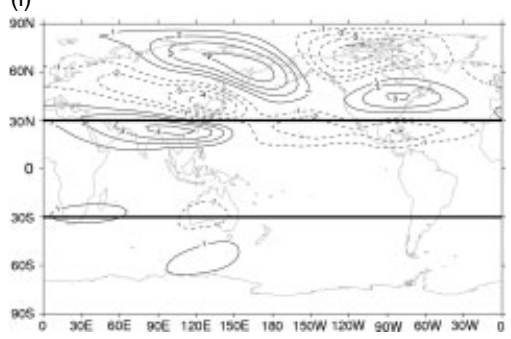

Figure 3. Streamfunction anomalies at $200 \mathrm{hPa}$ (units of $10^{6} \mathrm{~m}^{2} \mathrm{~s}^{-1}$ ) at day 30 obtained when the model is relaxed in the wider tropics $\left(30^{\circ} \mathrm{N}-30^{\circ} \mathrm{S}\right)($ the region relaxed is highlighted by the black solid lines) towards the response obtained to the heating over Indonesia (shown in Figure 2) with a relaxation time-scale of (a) $1 \mathrm{~h}$, (b) $10 \mathrm{~h}$, (c) 1 day and (d) 5 days. (e) -(h) are as (a) -(d) but for omega anomalies at $400 \mathrm{hPa}\left(\right.$ units of $2 \times 10^{-1} \mathrm{Pas}^{-1}$ ), whereas in (i) -(l) the temperature anomalies at $500 \mathrm{hPa}$ (units of $10^{-1} \mathrm{~K}$ ) are shown. The conventions are as in Figure 2.

ascent in the tropical heating region is about $1 / 3$ of the correct value for $10 \mathrm{~h}$ relaxation and its amplitude is fully reproduced for a time-scale of $1 \mathrm{~h}$. The wavetrain across the northern subtropical Pacific, however, is still well reproduced with time-scales up to 1 day. Away from the heating region, the accuracy of the results obtained for the temperature anomalies is similar to those obtained for the streamfunction anomalies, being well reproduced for time-scales up to 1 day. However, in the heating region the atmosphere is too cold for relaxation time-scales between $10 \mathrm{~h}$ and 5 days, but achieves approximately the correct value for a time-scale of $1 \mathrm{~h}$. 


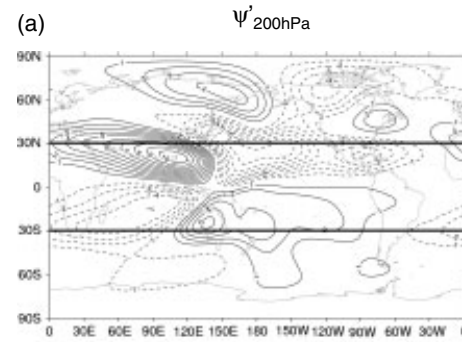

(d)

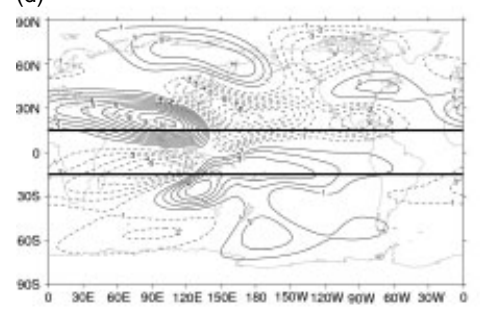

(g)

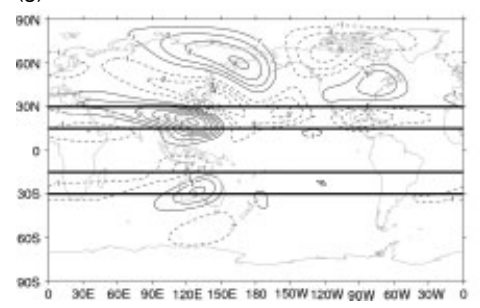

(b)

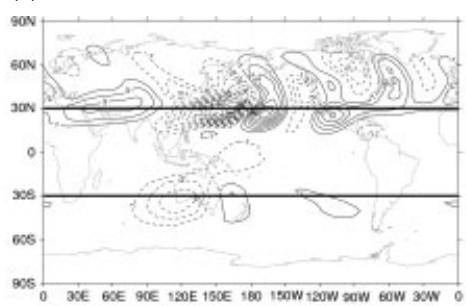

(e)

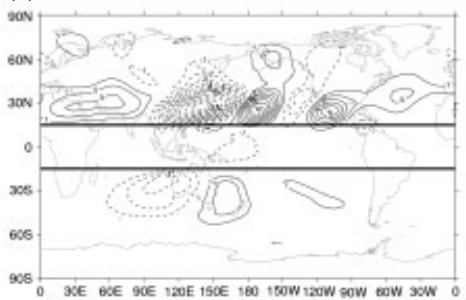

(h)

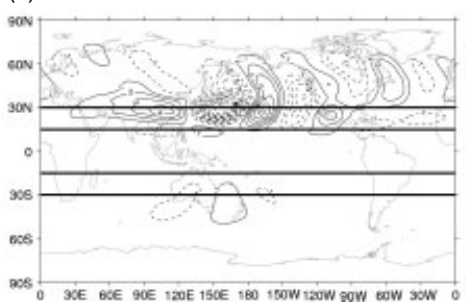

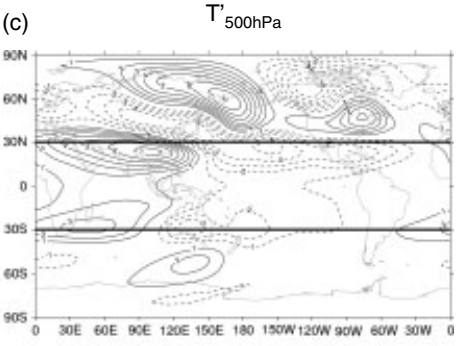

(f)

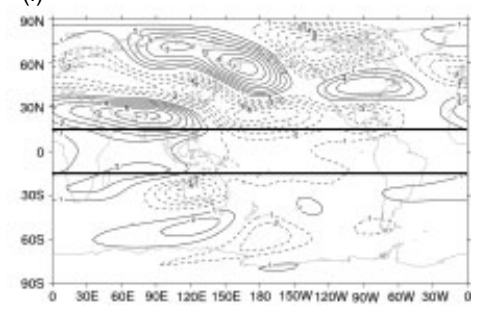

(i)

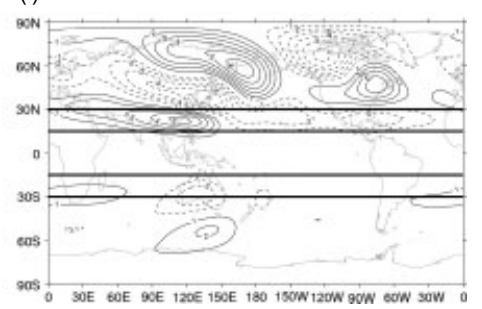

Figure 4. As Figure 3 but relaxing only the vorticity with a time-scale of $10 \mathrm{~h}$ in the wider tropics $\left(30^{\circ} \mathrm{N}-30^{\circ} \mathrm{S}\right)$ in $(\mathrm{a})-(\mathrm{c})$, deep tropics $\left(15^{\circ} \mathrm{N}-15^{\circ} \mathrm{S}\right)$ in (d) $-(\mathrm{f})$ and subtropics $\left(15^{\circ} \mathrm{N}-30^{\circ} \mathrm{N}+15^{\circ} \mathrm{S}-30^{\circ} \mathrm{S}\right)$ in $(\mathrm{g})-(\mathrm{i})$.

Figure 4 shows the results obtained when only the vorticity is relaxed in the wider tropics $\left(30^{\circ} \mathrm{S}-30^{\circ} \mathrm{N}\right)$, and also in the deep Tropics $\left(15^{\circ} \mathrm{S}-15^{\circ} \mathrm{N}\right)$ and subtropics $\left(15^{\circ} \mathrm{S}-30^{\circ} \mathrm{S}\right.$ and $15^{\circ} \mathrm{N}-30^{\circ} \mathrm{N}$ ), with a time-scale of $10 \mathrm{~h}$. It can be seen that relaxing the vorticity in the wider tropics gives responses in streamfunction and temperature that are almost identical to those obtained when all the model variables are relaxed in the same region. The tropical temperature anomalies are correctly given to be small, but their distribution is not accurate. The vertical velocity reproduces the subtropical wave train quite well, but completely misses the ascent in the heating region, as shown in Figure $4(\mathrm{~b})$. When the vorticity is relaxed only in the deep Tropics, the region in which the heating is situated, the results in Figures $4(\mathrm{~d})$ to $4(\mathrm{f})$ show that the streamfunction and temperature are still well reproduced. For subtropical forcing $\left(15^{\circ}-30^{\circ}\right.$ in both hemispheres) the tropical streamfunction and temperature, Figures $4(\mathrm{~g})$ to $4(\mathrm{i})$, have reduced amplitude but the extratropical structure is still well reproduced.

Figure 5 shows the results obtained when only the temperature is relaxed with $10 \mathrm{~h}$ time-scale. When it is relaxed in the wider tropics the anomalies are reproduced and there is even local ascent in the heating region although with much weaker amplitude and a rather different structure than that obtained when all the fields are relaxed. Apart from this, the phase and amplitude of the original response is not quite as well reproduced as when the vorticity is relaxed. This is particularly the case for the subtropical wave train in the omega and the temperature anomalies. When the temperature is relaxed in the deep Tropics the phase is still well captured but the amplitude is weaker, whereas when it is relaxed in the Subtropics there is an important phase difference over the North Pacific with the ridge over northeastern Russia being displaced to the east over western Alaska. However, in this experiment the amplitude is similar to that obtained when the field is relaxed in the wider tropics.

Figure 6 shows the model responses when only the divergence is relaxed using two different time-scales. When the wider tropics are relaxed with a time-scale of $10 \mathrm{~h}$, the phases of the responses in the streamfunction and temperature anomalies are reproduced but the amplitudes are in general much weaker (Figures 6(d) and 6(f)). The exception to this is the temperature anomaly in the wider region of the heating in the control, which becomes quite strongly negative. The Pacific wave train is also reproduced with much weaker amplitudes. The omega anomalies, Figure 6(e), are very weak and the local ascent in the heating region is only weakly reproduced. With a relaxation time-scale of $1 \mathrm{~h}$, however, all the fields are well simulated (Figures 6(a) to 6(c)) except that there is now a very large negative temperature error in the region of the heating in the control.

The response obtained when the logarithm of the surface pressure is relaxed towards the heating response in the tropics is negligible (not shown).

\section{Theory and analysis of model results}

It is useful to consider the basic balances in the equations for the case of idealized large-scale heating in the tropics, following Hoskins and Karoly (1981) and Hoskins (1987). Equations (1)-(5) are a Boussinesq, non-divergent set of equations written in terms of the variables $\psi^{\prime}, \phi^{\prime}, \theta^{\prime}, D^{\prime}$ and $w^{\prime}$ that denote perturbations in streamfunction, geopotential, potential temperature, divergence and vertical 


\section{B. Hoskins et al.}
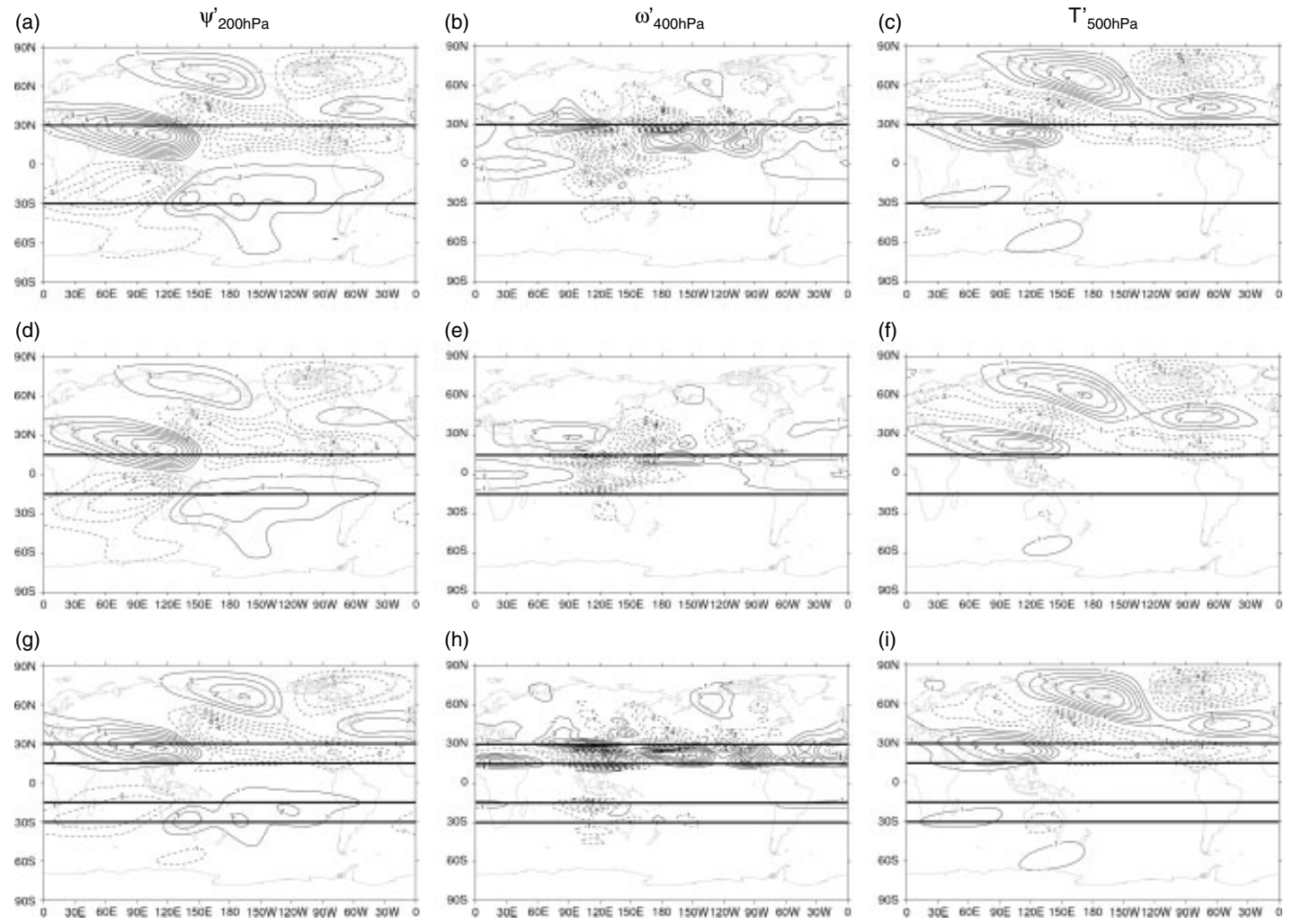

Figure 5. As Figure 4 but relaxing only the temperature.
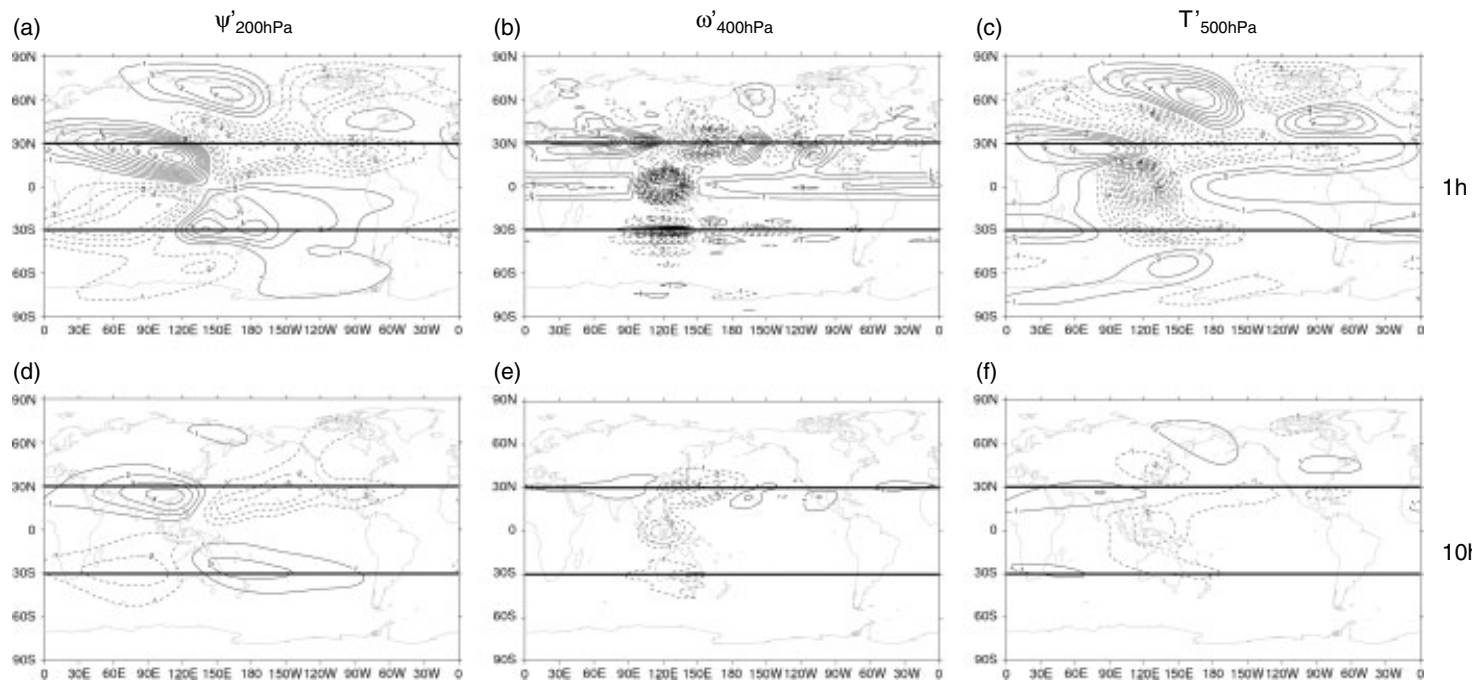

Figure 6. As Figure 3 but relaxing only the divergence in the wider tropics $\left(30^{\circ} \mathrm{N}-30^{\circ} \mathrm{S}\right)$ on a time-scale of $1 \mathrm{~h}$ in $(\mathrm{a})-(\mathrm{c})$ and $10 \mathrm{~h}$ in $(\mathrm{d})-(\mathrm{f})$.

velocity, respectively:

vorticity equation : $\beta \frac{\partial \psi}{\partial x}=-f D$

divergence equation : $\nabla^{2} \phi^{\prime}=\nabla \cdot\left(f \nabla \psi^{\prime}\right)$,

thermodynamic equation : $w^{\prime} \frac{\mathrm{d} \theta}{\mathrm{d} z}=Q$,

hydrostatic equation : $\frac{g}{\theta_{0}} \theta^{\prime}=\frac{\partial \theta^{\prime}}{\partial z}$,

continuity equation : $-D^{\prime}=\frac{\partial w^{\prime}}{\partial z}$,

where $\mathrm{d} \theta / \mathrm{d} z$ is a reference vertical gradient in potential temperature.
Note that Eq. (1) is the Sverdrup relation and Eq. (2) is the balance equation (Charney, 1955). With $Q$, the diabatic heating, specified there is a chain of deductions:

$$
Q \stackrel{(3)}{\rightarrow} w^{\prime} \stackrel{(5)}{\rightarrow} D^{\prime} \stackrel{(1)}{\rightarrow} \psi^{\prime} \stackrel{(2)}{\rightarrow} \phi^{\prime} \stackrel{(4)}{\rightarrow} \theta^{\prime} .
$$

It is of note that only the rotational flow is derived from its own prognostic equation, the vorticity equation. The other variables are all derived from balances in prognostic equations for other dependent variables. In particular, the (potential) temperature is at the far end of the chain of deductions from the specified heating, $Q$. It should be noted that all the links in the chain of deduction are two-way. In 
particular, if $\theta^{\prime}$ is specified then all the other variables in the chain and also $Q$ may in theory be deduced.

We now introduce scalings for all the dependent variables and also length scales in the zonal and meridional directions, $L_{X}$ and $L_{Y}$, respectively, a height scale $\boldsymbol{H}$, and typical values of $\mathbf{f}$ and its meridional gradient $\boldsymbol{\beta}$. Distinguishing between the zonal and meridional directions enables consideration of the aspect ratio, $\gamma=L_{X} / L_{Y}$, which is typically $>1$, i.e. zonal elongation. Also, it is reasonable to take $f=\boldsymbol{\beta} \boldsymbol{L}_{Y}$. However, for completeness we introduce $\alpha=\max \left(1, f / \beta L_{Y}\right)$ with the notion that typically $\boldsymbol{\beta}=\mathbf{1}$. Then Eqs (1)-(5) give:

$$
\begin{aligned}
\beta \frac{\psi}{L_{X}} & \sim f D \Leftrightarrow \psi \sim \frac{f L_{X}}{\beta} D, \\
\frac{\phi}{L_{Y}^{2}} & \sim \frac{\alpha \beta \psi}{L_{Y}} \Leftrightarrow \phi \sim \alpha \beta L_{Y} \psi, \\
w N^{2} & \sim \frac{g}{\theta_{0}} Q \Leftrightarrow w \sim \frac{1}{N^{2}} \frac{g}{\theta_{0}} Q, \\
\frac{g}{\theta_{0}} \theta & \sim \frac{\phi}{H} \Leftrightarrow \theta \sim \frac{\theta_{0}}{g H} \phi, \\
D & \sim \frac{w}{H},
\end{aligned}
$$

where $N^{2} \sim \frac{g}{\theta_{0}} \frac{\mathrm{d} \theta}{\mathrm{d} z}$ is the square of a Brunt-Väisälä (buoyancy) frequency.

Defining also a Burger number, $B \sim \frac{N^{2} H^{2}}{f^{2} L^{2}}$, it follows from Eqs (6)-(10) that

$$
\theta \sim \frac{\alpha}{B} \frac{1}{f} Q
$$

Taking typical values $f \sim 3 \cdot \mathbf{1 0}^{-5} \mathrm{~s}^{-1}, N \sim 1 \cdot 10^{-2} \mathrm{~s}^{-1}$, $L_{X} \sim 3 \cdot 10^{6} \mathrm{~m}$ and $L_{Y} \sim 1 \cdot 10^{6} \mathrm{~m}(\gamma \sim 1), H \sim 5 \cdot 10^{3} \mathrm{~m}$, then $\alpha \sim 1$ and $B \sim 1$ which implies that $\theta \sim \frac{1}{f} Q$. This means that the potential temperature scales as the amount of heating in time $\boldsymbol{f}^{-1} \sim \mathbf{0 . 4}$ days. For a heating of $5 \mathrm{~K} \mathrm{day}^{-1}$ this gives $\theta \sim 2 K$.

If a relaxation to a specified field or damping to zero is introduced in one of the equations it will have a significant impact only if it is at least comparable with the dominant terms in that equation. A scale analysis can then give the critical time-scale $\tau$ for such a relaxation or damping to be effective.

For significant damping in the vorticity $(\xi)$ equation, $\xi / \tau_{\xi} \sim \boldsymbol{\beta} \psi / L_{X}$, where $\tau_{\xi}$ is the critical time-scale for the vorticity. Using $\xi \sim \psi / L_{Y}^{2}$ gives

$$
\tau_{\xi} \sim \gamma \frac{1}{\beta L_{Y}} \sim \gamma \frac{f}{\beta L_{Y}} \frac{1}{f} .
$$

For damping to be significant in the divergence equation, $D / \tau_{D} \sim \nabla \cdot(f \nabla \psi) \sim \alpha f D \gamma$, where $\tau_{D}$ is the critical timescale for the divergence. Therefore, it can be concluded that

$$
\tau_{D} \sim \frac{1}{\alpha \gamma f} .
$$

Similarly for the thermodynamic equation $\theta / \tau_{\theta} \sim Q$. From Eq. (11) this gives

$$
\tau_{\theta} \sim \frac{\alpha}{B} \frac{1}{f} .
$$

Therefore the time-scale in each case is proportional to $f^{-1}$. However, for the typical numbers introduced above, the factors multiplying this are 3,1/3 and 1 for the vorticity, divergence and temperature equations, respectively. Using the value of $f$ then the critical time-scales are 1.2 days for the vorticity, 0.13 days $\sim 3 \mathrm{~h}$ for the divergence and 0.4 days $\sim 10 \mathrm{~h}$ for the temperature.

This theory can now be used to interpret the tropical component of the idealized experiments presented in section 3. Considering the experiment shown in Figure 4 with vorticity relaxation on a time-scale of $10 \mathrm{~h}$, as this is short compared with $\boldsymbol{\tau}_{\boldsymbol{\xi}}$ then it is to be expected that the tropical vorticity will be strongly relaxed towards the control distribution. This was indeed found in Figures 4(a) and 4(d). The last two elements of the chain of deduction suggest that the pressure and temperature fields will also be close to those in the control, in general agreement with Figures 4(c) and $4(\mathrm{f})$. However, the reverse chain to give $D^{\prime}$ and hence the vertical motion is broken by the strong additional relaxation term in the vorticity equation. Further, the lack of heating or relaxation terms in the thermodynamic equation means that the vertical motion will be negligible, consistent with the results in Figures 4(b) and 4(e).

The equivalent barotropic Rossby wave train that determines the far-field extratropical response depends on accurately capturing the early part of the wave train in the subtropical region. It is therefore consistent that all the experiments shown in Figure 4 capture well the extratropical response, including the vertical motion that is an integral component of an equivalent barotropic Rossby wave. Following Sardeshmukh and Hoskins (1988), it is important to represent outside the forcing region the Rossby wave source $R \boldsymbol{W} S=-\zeta \nabla \cdot V_{x}-V_{x} \cdot \nabla \zeta$, where $\zeta$ is the absolute vorticity and $V_{x}$ the divergent wind. The former will be well represented as part of the subtropical structure in all cases in Figure 4. Given the error in the tropical divergent wind field when only the vorticity is relaxed, the global $V_{\boldsymbol{x}}$ field and therefore the latter term will be in error. However, it is usually of importance only in the region of a strong localised jet stream, and is not significant here. As shown by Sardeshmukh and Hoskins (1988), it can be significant near strong subtropical jet streams, in which case this error could influence the correct representation of the Rossby wave trains in the extratropics.

Turning to the temperature relaxation experiments shown in Figure 5 the relaxation time-scale of $10 \mathrm{~h}$ is comparable to $\tau_{\theta}$ so that it would be expected that the tropical temperature field would resemble that in the control field but perhaps be not quite as accurate as those from the vorticity relaxation. Figures 5(a) and 5(c) are quite accurate but it is arguable that the results in Figures 4(a) and 4(c) for the vorticity relaxation are slightly better. The last four elements of the chain of deduction are reversible because they come from balances in the basic equations. Hence the expectation is that the tropical pressure, streamfunction, divergence and vertical motion fields will also be like the control fields but with reduced amplitude, consistent with the actual results in Figures 5(a) and 5(d), and Figures 5(b) and 5(e).

Comparing with vorticity and thermal relaxations on the same $10 \mathrm{~h}$ time-scale, the latter gives streamfunction and temperature fields of rather smaller magnitude. However, the tropical vertical velocity field is superior, although much reduced in magnitude compared with the control. With much stronger relaxation on a $1 \mathrm{~h}$ time-scale this 


\section{B. Hoskins et al.}

amplitude is approximately correct (not shown). With $10 \mathrm{~h}$ thermal relaxation, the extratropical wave train is slightly weaker, reflecting the weaker tropical rotational motion rather than the potentially superior divergent advection term in the RWS.

For relaxation of divergence, a $10 \mathrm{~h}$ time-scale is much longer than $\boldsymbol{\tau}_{D}$ and the very small amplitudes in Figures $6(\mathrm{~d})$ and $6(\mathrm{f})$ are consistent with this. However, $1 \mathrm{~h}$ is a short time-scale and the control divergence motion is obtained, Figure $6(\mathrm{~b})$. The chain of deduction provides that the streamfunction should also be well reproduced, consistent with Figure 6(a). However, the tropical balance in the divergence and thermodynamic equations is disrupted so that the pressure and temperature there will be in error. Even at $10 \mathrm{~h}$ relaxation, the ascent leads to significant cooling in the region of tropical heating in the control. With a $1 \mathrm{~h}$ time-scale this cooling error becomes extremely large.

It has been shown above that the tropical vertical velocity is reproduced for $1 \mathrm{~h}$ temperature or divergence relaxation. Therefore, in these cases the approximate thermodynamic equation balance (Eq. (3)), the first link in the chain of deduction, can be used to give a good estimate of the diabatic heating that led to the anomalies.

Finally, we consider experiments shown in Figure 2 with relaxation of all fields but with a range of time-scales. For a $1 \mathrm{~h}$ time-scale all the fields are strongly relaxed. For a $10 \mathrm{~h}$ relaxation the vorticity relaxation can be expected to dominate in reproducing the control fields, but the vertical motion relies on the thermal relaxation and is again reduced to about $30 \%$ of the magnitude in the control. Thus the divergent velocity term in the RWS will in general be reduced in magnitude, which could be an important error in some cases. Also an estimate of the tropical diabatic heating from the vertical motion would have a structure similar to that imposed in the control but about $30 \%$ of its magnitude. For 1 to 5 day relaxation streamfunction and temperature magnitudes become weaker as the vorticity relaxation in particular has less impact. The extratropical responses in each case reflect the varying amplitudes in the Subtropics.

\section{Conclusions}

In this paper a simple dynamical core model, the dynamical circulation model, has been used to investigate the technique of tropical relaxation and some theory has been developed to provide a basic theoretical framework for analysing the results.

First a case study of one anomalous 2-month period was presented. It was shown that one 30-day integration of the DCM relaxed to the mean analysed tropical state for this period produced a global pattern of anomalies with similar skill as a multimember ensemble using a state-ofthe-art forecast model. In fact the location of the low that was crucial for the anomalous UK weather in this season was better represented in the DCM. This suggested that the remarkable skill in reproducing the seasonal anomalies was because of their direct association with Rossby wave propagation from the tropics. However, the Northern Hemisphere amplitudes with the DCM were about half those in both the forecast model ensemble and in the analysis, which indicated amplification associated with the transient motions and physical processes simulated in the forecast model system. However, it also suggested that it was useful to use the DCM to examine the sensitivity of the tropical forcing technique to the various choices that could be made.

The theory described in section 4 is consistent with and supported by the set of DCM experimental results given in section 3. This theory suggests that if large-scale tropical diabatic heating is specified the basic equations become a set of diagnostic balances and give a chain of deductions for the variables in the tropical atmosphere. In this chain, the only equation that leads directly to the determination of the relevant prognostic variable is the vorticity equation.

Further it has been shown that the relaxation of tropical vorticity, temperature and divergence are important if the time-scales of relaxation are comparable or less than critical time-scales for that particular variable. These time-scales are all of order $f^{-1} \sim 10$ h. However, the non-dimensional factors for the large-scale tropics are such that these critical time-scales are typically 1.2 days for vorticity, $10 \mathrm{~h}$ for temperature and $3 \mathrm{~h}$ for divergence. Thus relaxation on a time-scale of the order of 1 day is most effective on the vorticity. This is the case even when the global flow that is to be reproduced is driven by tropical heating. The usual choice of $10 \mathrm{~h}$ made by ECMWF in their experiments is consistent with these time-scales, as is the skill in the reproduction of the June-July 2007 anomalies shown in Figure 1. In all the cases here, simulating an accurate rotational flow in the Subtropics leads to an accurate Rossby wave source and therefore accurate Rossby wave trains into the extratropics, with consequent accurate global anomaly patterns.

The chain of deduction of tropical variables is disrupted if the actual adiabatic heating is replaced by large relaxations terms in one or more equations. If only the vorticity is relaxed then the temperature and vertical motion anomalies in a tropical heating region will be very small. If temperature itself is relaxed on a short enough time-scale then the tropical structure of tropical temperature and vertical motion are both obtained. The vertical motion in a heating region in the tropics is also reproduced correctly if the divergence itself is strongly damped, i.e. with a time-scale of the order of $3 \mathrm{~h}$ or less. When the vertical motion is reproduced then the tropical diabatic heating itself can be estimated.

Since the aim of tropical relaxation experiments is usually to evaluate the impact on the extratropics, the Rossby wave propagation from a correctly simulated Subtropics is the crucial ingredient and these tropical errors will usually be irrelevant. A time-scale of $10 \mathrm{~h}$ on vorticity or on all variables is sufficient for this. However, near strong jet streams the error in the global divergent flow linked to the tropics could lead to a significant error in the divergent advection of vorticity term in the RWS and therefore to an error in the extratropical Rossby wave trains forced by tropical heating.

It is of interest to relate the critical time-scale for thermal relaxation, about 10 hours, to research on convective parametrizations. Some convection schemes lead to a heating term in the thermodynamic equation and others (adjustment schemes) to a relaxation term. In addition, the latter include a moisture relaxation, which also leads to a latent heating term. Frierson (2007), using an idealized model, found that there was little sensitivity to relaxation time-scales in an adjustment scheme for time-scales below a threshold of about $8 \mathrm{~h}$, consistent with the analysis presented here. Finally, we believe that the evidence given here suggests that the DCM with tropical relaxation could prove a very useful tool for informing and guiding experimentation with more complex models. 


\section{Acknowledgement}

This research was performed in 2007-2010 while the second author was doing a PhD at the Department of Meteorology, University of Reading and was sponsored by the Portuguese Foundation for Science and Technology (FCT) under the project SFRH/BD/36805/2007. Dr Paul Berrisford is gratefully acknowledged for making ERAInterim data available and easily accessible through the UKMO/BADC website. Special thanks are owed to Andy Heaps for his help with IDL in producing the plots.

\section{References}

Blackburn M, Methven J, Roberts N. 2008. Large-scale context for the UK floods in Summer 2007. Weather 63: 280-288.

Charney JG. 1955. The use of the primitive equations of motion in numerical preditciton. Tellus 7: 22-26.

Ferranti L, Palmer TN, Molteni F, Klinker E. 1990 Tropical-extratropical interaction associated with the 30-60 day oscillation and its impact on medium and extended range prediction. J. Atmos. Sci. 47: 2117-2199.

Frierson DMW. 2007. The dynamics of idealized convection schemes and their effect on the zonally averaged tropical circulation. J. Atmos. Sci. 64: 1959-1976.

Hoskins BJ. 1987. Diagnosis of forced and free variability in the atmosphere. Proceedings, RMS/AMS Conference on Atmospheric Variability on Seasonal and Longer Time Scales, London, September 1986.
Hoskins BJ, Karoly DJ. 1981. The steady linear response of a spherical atmosphere to thermal and orographic forcing. J. Atmos. Sci. 38: 1179-1196.

Hoskins BJ, Simmons A. 1975. A multi-layer spectral model and the semi-implicit method. Q. J. R. Meteorol. Soc. 101: 637-655.

Jin F-F, Hoskins BJ. 1995. The direct response to tropical heating in a baroclinic atmosphere. J. Atmos. Sci. 52: 307-319.

Jung T. 2011. Diagnosing remote origins of forecast error: relaxation versus 4D-Var data-assimilation experiments. Q. J. R. Meteorol. Soc. 137: $598-606$

Jung T, Palmer T, Rodwell M, Serrar S. 2008. Diagnosing forecast error using relaxation experiments. ECMWF Newsletter 116. ECMWF: Shinfield Park, Reading, Berkshire, UK. Available at http://www.ecmwf.int/publications/

Jung T, Miller M, Palmer T. 2010a. Diagnosing the origin of extendedrange forecast errors. Mon. Weath. Rev. 138: 2434-2446.

Jung T, Miller M, Palmer T. 2010b. Understanding the anomalously cold European winter of 2005/2006 using relaxation experiments. Mon. Weath. Rev. 138: 3157-3174.

Klinker E. 1990. Investigation of systematic errors by relaxation experiments. Q. J. R. Meteorol. Soc. 116: 573-594.

Pohl B, Douville H 2011. Diagnosing GCM errors over West Africa using relaxation experiments. Part I: summer monsoon climatology and interannual variability. Clim. Dyn. 37: 1293-1312, DOI: 10.1007/s00382-010-0911-2.

Rodwell MJ, Hoskins BJ. 1995. A model of the Asian Summer Monsoon Part II: Cross-equatorial flow and PV behavior. J. Atmos. Sci. 52: 1341-1356.

Sardeshmukh PD, Hoskins BJ. 1988. Generation of global rotational flow by steady idealized tropical divergence. J. Atmos. Sci. 45: 1228-1251.

Vitart F, Jung T. 2010. Impact of the Northern Hemisphere extratropics on the skill in predicting the Madden Julian Oscillation. Geophys. Res. Lett. 37: L23805. 